
\title{
$\begin{array}{ll}\text { Research Square } & \begin{array}{l}\text { Preprints are preliminary reports that have not undergone peer review. } \\ \text { They should not be considered conclusive, used to inform clinical practice, } \\ \text { or referenced by the media as validated information. }\end{array}\end{array}$
}

\section{Multi-Photon Microscopy for the Evaluation of Interstitial Fibrosis in Extended Criteria Donor Kidneys: A Proof-of-Concept Study}

\author{
Wei Zheng So \\ Yong Loo Lin School of Medicine, National University Singapore https://orcid.org/0000-0002-2303-0750 \\ Rachel Zui Chih Teo \\ Division of Nephrology, Department of Medicine, National University Hospital, Singapore \\ Li Yin Ooi \\ Department of Pathology, National University Hospital, Singapore \\ Benjamin Yen Seow Goh \\ Department of Urology, National University Hospital, Singapore \\ Jirong Lu \\ Department of Urology, National University Hospital, Singapore \\ Anantharaman Vathsala \\ Division of Nephrology, Department of Medicine, National University Hospital, Singapore \\ Thomas Paulraj Thamboo \\ Department of Pathology, National University Hospital, Singapore \\ Ho Yee Tiong ( $\nabla$ surthy@nus.edu.sg) \\ Department of Urology, National University Hospital, Singapore https://orcid.org/0000-0003-0077-7904
}

\section{Research Article}

Keywords: multiphoton microscopy, extended criteria donor, kidney transplantation

Posted Date: November 19th, 2021

DOI: https://doi.org/10.21203/rs.3.rs-1093197/v2

License: (ㅇ) (i) This work is licensed under a Creative Commons Attribution 4.0 International License. Read Full License 


\section{Abstract}

Introduction:

This study evaluated the initial use of label-free second harmonic generation (SHG) imaging with two-photon excitation (2PE) autofluorescence for the quantification of collagen/fibrosis on pre-implantation biopsies of extended criteria donors (ECD).

\section{Methods:}

A total of 20 core ECD kidney transplant biopsy specimen tissues from 7 ECD donors, taken at the time of pre-implantation were retrieved, cut into 5-micron sections, mounted on slides and deparaffinized. The core needle biopsies were imaged with $2 X$ and $20 X$ objective using the commercially available laser-based Genesis® 200 (Histoindex Pte Ltd and Clinnovate Pte Ltd, Singapore). The entire core was selected as the Region of Interest (ROI). Corresponding clinical information from transplant donors were retrieved. Histopathological review was performed, and all biopsies had Interstitial Fibrosis (IF)/Tubular Atrophy (TA) scores $>0$. Collagen parameters measured included quantification by the Collagen Area Ratio in Tissue (CART) and qualitative measurements by Collagen Reticulation Index (CRI).

\section{Results:}

20 explant core biopsies were extracted from 10 donor kidney samples, of which originated from 7 donors. Table 1 depicts the baseline ECD characteristics of the donors. For the kidneys with multiple biopsies done, we obtained an average score of the collagen parameters across the different samples. Biopsies classified with $>85 \% \mathrm{KDPI}$ score had significantly higher CAR and CART than biopsies with $\leq 85 \%$ KDPI score.

\section{Conclusion:}

MPM is an evolving technology that enables the quantification of the amount (CART) and quality (CRI) of collagen deposition in unstained explant biopsies of ECD kidneys. This initial evaluation found significant differences in both parameters between ECD kidneys with more or less than $85 \% \mathrm{KDPI}$ scores.

\section{Introduction}

Renal anatomy and intrinsic residual function have always been of paramount importance in stratifying deceased donor kidneys for transplantation. This is particularly so for extended criteria donors (ECD) who do not fulfil the criteria of a standard criteria donor (SCD). Given that ECD kidneys have a reportedly $70 \%$ higher risk of graft failure than non-ECD kidneys ${ }^{1}$, it is imperative to appropriately risk-stratify across the spectrum of available ECDs. Pertinently, the dichotomous classification of single versus dual implantation has also reaffirmed the need to objectively characterize the quality of a donor kidney².

One commonly utilized method to do so is the procurement of a pre-implantation renal biopsy for histopathological analysis. Remuzzi et al. pioneered a solely histological-based selection criteria to evaluate the quality of ECD kidneys and henceforth classify them as a single or dual implant, utilizing four variables to distinguish - glomerular global sclerosis, tubular atrophy, interstitial fibrosis, and arterial and arteriolar narrowing ${ }^{3}$. On the other hand, frameworks such as the Kidney Donor Profile Index (KDPI) developed by the United Network for Organ Sharing Kidney Transplantation Committee ${ }^{4}$ aim to incorporate relevant clinical parameters as adjuncts to complement the SCD/ECD classification. However, it is worth noting that KDPI was solely derived from Western transplant registries, thereby alluding to a possibly unvalidated usage if utilized beyond the United States.

As a transplant center located within Asia, we previously sought to clarify whether clinical evaluation by KDPI was superior to histopathological assessment of ECD kidneys ${ }^{5}$. It was concluded that KDPI per se was insufficient to stratify single versus dual ECD 
implants, and that Remuzzi scoring demonstrated higher sensitivity, specificity, and positive predictive value in selecting kidneys for dual implants. Patients with KDPI > 1.1 were recommended still have routine biopsies to optimize characterization of ECD kidneys.

In line with the advancements of in vivo histological techniques, growing interest has centered around multiphoton microscopy (MPM) as a step up from conventional staining methods ${ }^{6}$. It combines Second Harmonic Generation (SHG) and Two Photon Excitation (TPE) Fluorescence to image extracellular matrix architecture in intricate detail ${ }^{7}$. MPM has been previously utilized for deep optical sectioning of live human tissue and is exceptionally accurate at demonstrating specific contrast in tissue exhibiting fibrillar collagenous qualities ${ }^{8}$.

Taken within the context of ECD classification, our interest has been drawn to assessing its predictive ability in the quantification of renal interstitial fibrosis. Since it comprises part of Remuzzi's variables, the aim of the present study was to employ MPM as a novel imaging technique to characterize and quantify interstitial fibrosis in kidney explant biopsies, assessing for any correlation between its parameters and recipient outcomes post-transplant.

\section{Methods}

\section{Sample Retrieval}

The study protocol was approved by the National Healthcare Group Domain Specific Review Board, Reference Number: 2017/01003. Specific to our objectives, all explant core biopsy samples were extracted from deceased donors that fulfilled the Extended Criteria Donor (ECD) standards, as delineated by the Scientific Registry of Transplant Recipients. This is defined as (1) kidneys retrieved from donors aged 60 years or above, or (2) donors aged 50 to 59 years old with at least two of the following: (3) serum creatinine > $1.5 \mathrm{mg} / \mathrm{dL}$, (4) history of hypertension, or (5) death resulting from a cerebrovascular accident. From the Ethics Board-approved tissue repository, 22 core ECD kidney transplant biopsy specimen tissue blocks from our Centre (National University Hospital, Singapore) were selected and retrieved from 7 ECD donors. All 22 specimens were taken at the time of pre-implantation. Qualities of all deceaseddonor kidneys were evaluated by an experienced pathologist, classifying the samples according to the Remuzzi histological scoring system. The biopsy samples were correspondingly allocated an Interstitial Fibrosis score for the purposes of analysis. Nonidentifiable corresponding clinical information from our kidney transplant centre database was retrieved.

\section{Sample Preparation and Multi-Photon Microscopy}

The unstained de-paraffinized Formalin-Fixed Paraffin-Embedded tissues of 5-micron thickness were imaged using the commercially available laser-based Genesis200 Multi-Photon Microscopy system (Histoindex Pte. Ltd, Singapore). Image acquisition was performed at $2 \mathrm{X}$ and $20 \mathrm{X}$ objective with $512 \times 512$-pixel resolution. MPM is an imaging modality comprising SHG and TPE microscopy. SHG signals are detected when a laser of femtosecond range interacts with tissues that have unique, noncentrosymmetric structure within the ECM such as collagen fibers and elastin. When this SHG signal is combined with autofluorescence signal from the cells using TPE, MPM is able to penetrate deep into tissues to generate a high-resolution image that reflects both the architecture of the ECM and surrounding cellular structure. On SHG microscopy, the fibrosis within the renal interstitium was readily identified, with fluorescent green signals on SHG analysis corresponding to collagen fibers. In contrast, collagen-devoid tumor tissues and collagen-poor normal renal parenchyma demonstrated minimal SHG signals.

Embedded within the proprietary stain-free imaging technology and analysis software has the capability to detect sensitively and quantify fine collagen dynamics that are otherwise unobserved with traditional staining techniques. The analysis is able to identify and characterize previously described SHG collagen characteristic that corresponds with the collagen profiles of the tissues. The percentage of collagen in relation to the total region of interest is defined as the Collagen Area Ratio (CAR). The percentage of collagen within the total tissue sample was expressed as the Collagen Area Ratio in Total Tissue (CART). Both CAR and CART quantify the collagen content of the tissues. After the collagen area was defined, a skeletonization of the collagen was applied, providing a schematic representation of the collagen fibers and their intersection points. The Collagen Reticulation Index (CRI) was defined as the total number of intersection points in the given collagen area, representing the degree of reticulation and interconnection of collagen fibers.

Multi-photon imaging was performed and designated Regions of Interests (ROI) for analysis of collagen parameters in each specimen. At each region, clinically validated algorithms quantify collagen content and structure. These algorithms were validated 
against conventional stained Hematoxylin \& Eosin (H\&E) images by pathologists at the National University Hospital, Singapore (Figure 1 and 2). Collagen content was measured quantitatively by CAR and CART, and collagen structure was measured by CRI.

\section{Statistical Analysis}

Parametric continuous variables were presented as Mean +/-Standard Deviation (SD). Non-parametric continuous variables were reported as Median and Range. Data There were no missing values from data collected. Chi-squared tests (or Fisher's Exact Test, wherever applicable) were utilized to compare categorical variables. The Mann-Whitney U test was used to compare non-parametric continuous variables. Statistical significance in this study was determined as $P<0.05$. All reported $P$ values were 2 -sided, and analyses were performed with IBM SPSS Version 26.0.

\section{Results}

\section{Donor Baseline Characteristics}

Twenty explant core biopsies were imaged from 10 donor kidney samples, of which originated from 7 donors (Table 1). Baseline characteristics are detailed in Table 2. Mean donor age was 54.45 years $+/-7.95$, and $57.1 \%$ of the donors were male. 3 donors underwent single kidney implantation. The donors had a mean terminal creatinine level of $91.14 \mathrm{umol} / \mathrm{L}+/-30.11$ prior to transplantation. 16 core biopsies were allocated a Remuzzi scoring of 4 to 6, while the remaining 4 were categorized into the 0-3 group. Median Kidney Donor Profile Index (KPDI) scores across all donors was 76\% (IQR: 65.25 - 89\%), with 3 out of 7 donors obtaining a KDPI of more than $85 \%$. 
Table 1

Clinical, Histopathological and MPM-derived Collagen Parameters of 20 Explant Core Biopsy Samples

\begin{tabular}{|c|c|c|c|c|c|c|c|c|c|c|}
\hline Patient & $\begin{array}{l}\text { Biopsy } \\
\text { S/N }\end{array}$ & $\begin{array}{l}\text { Donor } \\
\text { Age }\end{array}$ & $\begin{array}{l}\text { Donor } \\
\text { Gender }\end{array}$ & $\begin{array}{l}\text { Donor } \\
\text { Hypertension }\end{array}$ & $\begin{array}{l}\text { Terminal } \\
\text { Creatinine } \\
\text { (umol/L) }\end{array}$ & $\begin{array}{l}\text { Kidney } \\
\text { Donor } \\
\text { Profile } \\
\text { Index } \\
\text { (KDPI) } \\
\text { Score }\end{array}$ & $\begin{array}{l}\text { Collagen } \\
\text { Area } \\
\text { Ratio } \\
\text { (CAR) }\end{array}$ & $\begin{array}{l}\text { Collagen } \\
\text { Area } \\
\text { Ratio in } \\
\text { Tissue } \\
\text { (CART) }\end{array}$ & $\begin{array}{l}\text { Collagen } \\
\text { Reticulation } \\
\text { Index (CRI) }\end{array}$ & $\begin{array}{l}\text { Remuzzi } \\
\text { Score }\end{array}$ \\
\hline \multirow[t]{2}{*}{ A } & 1 & 54.07 & Female & Yes & 58 & $72 \%$ & 33.50 & 50.44 & 3.90 & 5 \\
\hline & 2 & & & & & & 37.77 & 50.29 & 3.75 & 5 \\
\hline \multirow[t]{2}{*}{ B } & 3 & 57.55 & Female & No & 95 & $76 \%$ & 36.35 & 45.91 & 4.44 & 3 \\
\hline & 4 & & & & & & 39.01 & 51.40 & 4.40 & 3 \\
\hline \multirow[t]{4}{*}{ C } & 5 & 44.98 & Male & Yes & 93 & $63 \%$ & 22.13 & 32.21 & 4.77 & 4 \\
\hline & 6 & & & & & & 29.60 & 38.47 & 4.89 & 4 \\
\hline & 7 & & & & & & 30.16 & 38.85 & 4.78 & 3 \\
\hline & 8 & & & & & & 35.57 & 45.31 & 5.12 & 3 \\
\hline \multirow[t]{2}{*}{ D } & 9 & 56.91 & Female & Yes & 68 & $92 \%$ & 35.86 & 60.90 & 4.15 & 5 \\
\hline & 10 & & & & & & 31.33 & 46.59 & 4.15 & 5 \\
\hline \multirow[t]{4}{*}{$E$} & 11 & 58.55 & Male & Yes & 82 & $86 \%$ & 35.06 & 50.62 & 4.10 & 4 \\
\hline & 12 & & & & & & 34.46 & 51.72 & 4.34 & 4 \\
\hline & 13 & & & & & & 31.89 & 47.52 & 4.30 & 4 \\
\hline & 14 & & & & & & 36.30 & 52.15 & 4.70 & 4 \\
\hline \multirow[t]{4}{*}{$\mathrm{F}$} & 15 & 43.23 & Male & No & 152 & $46 \%$ & 36.78 & 53.50 & 4.53 & 5 \\
\hline & 16 & & & & & & 25.22 & 34.58 & 4.29 & 5 \\
\hline & 17 & & & & & & 39.13 & 51.01 & 5.31 & 4 \\
\hline & 18 & & & & & & 29.11 & 41.58 & 4.54 & 4 \\
\hline \multirow[t]{2}{*}{ G } & 19 & 65.88 & Male & Yes & 90 & $90 \%$ & 34.93 & 55.74 & 3.71 & 4 \\
\hline & 20 & & & & & & 34.97 & 56.80 & 3.62 & 4 \\
\hline
\end{tabular}


Table 2

ECD Baseline Characteristics

\begin{tabular}{|c|c|}
\hline & ECDs \\
\hline & $(n=7)$ \\
\hline Donor age, yr [mean (SD)] & $54.45(7.95)$ \\
\hline \multicolumn{2}{|l|}{ Gender [n (\%)] } \\
\hline Male & $4(57.1)$ \\
\hline Female & $3(42.9)$ \\
\hline \multicolumn{2}{|l|}{ Diabetes [n (\%)] } \\
\hline No & $5(71.4)$ \\
\hline Yes & $2(28.6)$ \\
\hline \multicolumn{2}{|l|}{ Hypertension [n (\%)] } \\
\hline No & $2(28.6)$ \\
\hline Yes & $5(71.4)$ \\
\hline \multicolumn{2}{|l|}{ Dialysis [n (\%)] } \\
\hline No & $7(100)$ \\
\hline Yes & $0(0)$ \\
\hline \multicolumn{2}{|l|}{ Acute Kidney Injury [n (\%)] } \\
\hline No & $5(71.4)$ \\
\hline Yes & $2(28.6)$ \\
\hline Terminal Creatinine (umol/L) [mean (SD)] & $91.14(30.11)$ \\
\hline \multicolumn{2}{|l|}{ Cause of Death [n (\%)] } \\
\hline Cerebrovascular Accident (Stroke) & $7(100)$ \\
\hline Others & $0(0)$ \\
\hline \multicolumn{2}{|l|}{ Remuzzi Score [n (\%)] } \\
\hline $0-3$ & $1(14.3)$ \\
\hline $4-6$ & $6(85.7)$ \\
\hline \multicolumn{2}{|l|}{ Remuzzi Score (No. of biopsies) [n (\%)] } \\
\hline $0-3$ & $4(20)$ \\
\hline $4-6$ & $16(80)$ \\
\hline Kidney Donor Profile Index (KDPI) Score \% [mean (SD)] & 75 (16.48) \\
\hline \multicolumn{2}{|l|}{ KDPI [n (\%)] } \\
\hline $20-85 \%$ & $4(57.1)$ \\
\hline$>85 \%$ & $3(42.9)$ \\
\hline
\end{tabular}

\section{Comparison of MPM-evaluated Collagen Characteristics by KDPI}

Although CAR values were unequivocally identical across all samples, biopsies classified with $>85 \% \mathrm{KDPI}$ demonstrated a significantly higher CART (51.94 vs. $45.61 ; p=0.011$ ) compared to biopsies with $20-85 \%$ KDPI percentages (Table 3 ). Conversely, they had lower CRI compared to biopsies with 20-85\% KDPI scores (4.15 vs. 4.53; $p=0.025$ ). 


\section{Comparison of Recipient Clinical Outcomes Post-Transplant by KDPI}

Recipient creatinine and eGFR levels at 1 and 3-years post-transplant did not differ significantly between 20-85\% and >85\% KDPI scores (Table 4). However, it is worth noting that single versus dual implantations may potentially contribute to further disparities in clinical outcomes - dual implant kidneys may have overcome the underlying deficiencies of donor kidneys with $>85 \% \mathrm{KDPI}$ scores. This precludes a definite comparison from being made.

\section{MPM-evaluated Collagen Parameters and Predictors of Long-term Recipient Outcomes}

Upon univariable analysis, CAR and CART were not observed to be independent predictors of recipient renal function at 1 and 3-years post-transplant. Interestingly, an increase in CRI correlated significantly with rise in recipient creatinine levels at 1-year post-transplant $(p=0.027 ; 95 \% \mathrm{Cl}: 4.635-66.797)$, demonstrating itself as an independent predictor of creatinine levels 1-year after transplantation.

Table 3

Comparison of CAR/CART/CRI by KDPI

\begin{tabular}{|llll|}
\hline & $\mathbf{2 0 - 8 5 \%}$ & $\mathbf{> 8 5 \%}$ & p-value \\
\cline { 2 - 4 } & $\mathbf{( n = 1 2 )}$ & $\mathbf{( n = 8 )}$ & \\
\hline Collagen Area Ratio (CAR) [median (IQR)] & $34.53(29.23-37.52)$ & $34.95(32.53-35.66)$ & 1.000 \\
\hline Collagen Area Ratio in Tissue (CART) [median (IQR)] & $45.61(38.56-50.87)$ & $51.94(48.29-56.54)$ & $\mathbf{0 . 0 1 1}$ \\
\hline Collagen Reticulation Index (CRI) [median (IQR)] & $4.53(4.31-4.86)$ & $4.15(3.80-4.33)$ & $\mathbf{0 . 0 2 5}$ \\
\hline
\end{tabular}

Table 4

Comparison of Clinical Outcomes by KDPI

\begin{tabular}{|llll|}
\hline & $\mathbf{2 0 - 8 5 \%}$ & $\mathbf{> 8 5 \%}$ & \multirow{2}{*}{-value } \\
\cline { 2 - 3 } & $\mathbf{( n = 1 2 )}$ & $(\mathbf{n = 8})$ & \\
\hline eGFR 1 Year (ml/min/1.73m²) [median (IQR)] & $68.50(43-105)$ & $53(12.75-55)$ & 0.160 \\
\hline Creatinine 1 Year (umol/L) [median (IQR)] & $93.50(54-168)$ & $105(26-106)$ & 0.348 \\
\hline eGFR 3 Years (ml/min/1.73m²) [median (IQR)] & $60(46-90)$ & $49.50(8.25-66)$ & 0.086 \\
\hline
\end{tabular}

\section{Discussion}

This proof-of-concept study demonstrated the use of commercially available MPM-enabled detailed imaging of renal interstitial fibrosis in explant core needle biopsies of ECD kidneys. CART and CRI values differed significantly between ECD kidneys stratified by KDPI scores. CRI was demonstrated to be a statistically significant predictor of recipient creatinine levels 1-year post-transplant.

Multi-photon technology flaunts in its ability to precisely quantify the extent of collagen tissue present in tissue, well going beyond to analyze collagen organization from the molecular up till the tissue architectural scale. This relies on its non-linear microscopic method of imaging ${ }^{9}$, as well as the involvement of the Second Harmonic Generation microscopy ${ }^{7}$. SHG allows for accurate identification of noncentrosymmetric molecules such as fibrillar collagens, which are unambiguously implicated in multi-system pathologies that demonstrate fibrosis ${ }^{10-14}$. Of which includes fibrillar collagen, that is found within the extracellular matrix of the renal parenchyma. This is also coupled with TPE - fluorescence imaging of the fibrotic regions, undergoing extrinsic fluorescent labeling and auto-fluorescence with two-photon excitation sequence ${ }^{15}$. In fact, MPM is similarly evaluated within the fields of genitourinary oncology, yielding some promising early results. It could potentially serve as a biomarker for prostate tumor aggressiveness ${ }^{16}$, or even as a prognostic indicator of progression of non-muscle invasive bladder cancer ${ }^{17}$.

Most notably, a distinct advantage that it has over routine histological assessment is the ability to evaluate tissue samples without the need for any staining ${ }^{7}$. Regarding histopathological analysis of kidney biopsies, pathologists have conventionally relied upon various methods such as light microscopy, immunohistochemistry and electron microscopy for the fixation, processing, sectioning 
and staining of a said tissue specimen ${ }^{18}$. Glomerulosclerosis, tubular atrophy, interstitial fibrosis, and vascular attenuation are the key points of interest to determine the classification, suitability, and type of transplantation to be performed. Given this knowledge, the relevance of MPM as an adjunct to histopathological or clinical frameworks sets a novel precedent for enhanced deceased donor kidney assessment. The ability of MPM to facilitate automated evaluation of the degree of renal interstitial fibrosis heralds the prospects of artificial intelligence within the context of histopathology evaluation ${ }^{19}$. Ongoing research to establish the utility of MPM and artificial intelligence in assessing the other components of Remuzzi scoring is underway ${ }^{20}$.

Ranjit et al. incorporated the use of SHG and fluorescence lifetime imaging (FLIM) of tissue autofluorescence as practical and unique method of quantifying renal fibrosis in fixed tissue samples of a mouse UUO model ${ }^{21}$. This was carried out via a deep tissue-imaging (Deep Imaging Via Enhanced-Photon Recovery (DIVER)) microscope. A classical experimental model of a unilateral ureteric obstruction was adopted for the system to quantify the extent of fibrosis present, reporting sensitivity and specificity values that were on-par with standard histological methods. Though a key difference is in the mode of fluorescent imaging used - FLIM, unlike twophoton excitation auto-fluorescence, does not depend on fluorescent intensity. Rather, it relies on the rate of decay of the fluorescent signal, postulating as a more specific approach to capture tissue autofluorescence. This suggests a possibility of an inferiority in TPE compared to FLIM in detecting collagenous tissue. The authors found that this combined SHG-FLIM method independently predicted the extent of fibrosis in the mouse model and concluded by advocating for co-existing usage of SHG-FLIM with pre-existing histological methods to augment the accuracy of collagen quantification.

To our knowledge, this is the first study to comparatively analyze MPM (combined SHG-TPE) technology in ECD kidneys with existing validated Remuzzi and KDPI criteria. As a proof-of-concept, the MPM-derived collagen quantifiers have been observed to have internal homogeneity in its evaluation of interstitial fibrosis across all 20 kidney biopsies. Scores were also consistent between biopsies from different kidneys that originated from the same donor. However, there was no accordant correlations between the collagen parameters and Remuzzi scoring, as well as KDPI values in all biopsy specimens.

\section{Limitations in Utility of MPM for Assessment for ECD Kidney Allocation}

There are some limitations to the present study. First, the sample size is small, affecting the validity and increasing the margin of error of the aforementioned results. Secondly, this study is retrospective in nature and hence prone to selection bias. A larger number over a longer duration of the study period would allow us to correlate MPM-derived collagen characteristics with more confidence. Lastly, although CRI was a statistically significant predictor of recipient creatinine levels 1 -year post-transplant, it should be noted that this is accompanied by correspondingly wide confidence intervals. Hence, concluding that CRI was truly indeed an independent predictor should be validated with future studies.

\section{Conclusion}

In this proof-of-concept study, we reported noteworthy differences in MPM-derived collagen parameters between ECD kidneys of varying KDPI scores. When pegged against validated histological and clinical frameworks in stratifying ECD kidney biopsies, it is still an evolving technology in question. With further advancements in imaging techniques, future observational studies are eagerly awaited to support or challenge the reproducibility of our findings.

\section{Declarations}

\section{Conflict of Interest:}

The authors declare that they have no conflict of interest.

\section{Financial Support:}

National University Health System Innovation Seed Grant 2017 (intra-institution). There was no extra-institution or commercial funding. 
Ethics Approval:

The study protocol was approved by the National Healthcare Group Domain Specific Review Board, Reference Number: 2017/01003.

\section{Author Contributions:}

WZS was involved in data analysis and manuscript writing. RZCT and LYO were involved the histological preparation of the samples. BYSG, JL, AV, TPT, HYT contributed to the conceptualization, planning and supervision of the study.

\section{Acknowledgements:}

The authors would like to acknowledge Histoindex Pte. Ltd, Singapore and Clinnovate Health Pte. Ltd, Singapore for performing Multiphoton Microscopy of the samples using the Genesis $200^{\text {TM }}$ system.

\section{References}

1. Port FK, Bragg-Gresham JL, Metzger RA, et al. Donor characteristics associated with reduced graft survival: an approach to expanding the pool of kidney donors1. Transplantation. 2002;74:1281-1286.

2. Khalil MAM, Tan J, Khan TFT, Khalil MAU, Azmat R. Dual Kidney Transplantation: A Review of Past and Prospect for Future. Int Sch Res Notices. 2017;2017:2693681-2693681.

3. Remuzzi G, Cravedi P, Perna A, et al. Long-term outcome of renal transplantation from older donors. New England Journal of Medicine. 2006;354:343-352.

4. Rao PS, Schaubel DE, Guidinger MK, et al. A comprehensive risk quantification score for deceased donor kidneys: the kidney donor risk index. Transplantation. 2009;88:231-236.

5. Vathsala A, Sran H, Tiong H-Y, et al. Explant biopsy and Remuzzi scoring outperforms Kidney Donor Profile Index (KDPI) in selection of extended criteria donor kidneys for single implant. Transplantation. 2018;102:S401.

6. Peti-Peterdi J, Kidokoro K, Riquier-Brison A. Novel in vivo techniques to visualize kidney anatomy and function. Kidney International. 2015;88:44-51.

7. Cicchi R, Vogler N, Kapsokalyvas D, Dietzek B, Popp J, Pavone FS. From molecular structure to tissue architecture: collagen organization probed by SHG microscopy. Journal of Biophotonics. 2013;6:129-142.

8. Peti-Peterdi J. A practical new way to measure kidney fibrosis. Kidney International. 2016;90:941-942.

9. Dunn KW, Young PA. Principles of multiphoton microscopy. Nephron Experimental Nephrology. 2006;103:e33-e40.

10. Ochoa LF, Kholodnykh A, Villarreal P, et al. Imaging of Murine Whole Lung Fibrosis by Large Scale 3D Microscopy aided by Tissue Optical Clearing. Scientific Reports. 2018;8:13348.

11. Strupler M, Pena A-M, Hernest M, et al. Second harmonic imaging and scoring of collagen in fibrotic tissues. Optics express. 2007;15:4054-4065.

12. Tai DC, Tan N, Xu S, et al. Fibro-C-Index: comprehensive, morphology-based quantification of liver fibrosis using second harmonic generation and two-photon microscopy. Journal of biomedical optics. 2009;14:044013.

13. Sun W, Chang S, Tai DC, et al. Nonlinear optical microscopy: use of second harmonic generation and two-photon microscopy for automated quantitative liver fibrosis studies. Journal of biomedical optics. 2008;13:064010.

14. Peng Q, Zhuo S, So PT, Yu H. Improving liver fibrosis diagnosis based on forward and backward second harmonic generation signals. Applied Physics Letters. 2015;106:083701.

15. Stanciu SG, Xu S, Peng Q, et al. Experimenting liver fibrosis diagnostic by two photon excitation microscopy and bag-of-features image classification. Scientific reports. 2014;4:1-12.

16. Ling Y, Li C, Feng K, et al. Second harmonic generation (SHG) imaging of cancer heterogeneity in ultrasound guided biopsies of prostate in men suspected with prostate cancer. Journal of biophotonics. 2017;10:911-918. 
17. Brooks M, Mo Q, Krasnow R, et al. Positive association of collagen type I with non-muscle invasive bladder cancer progression. Oncotarget. 2016;7:82609.

18. Walker PD, Cavallo T, Bonsib SM, The Ad Hoc Committee on Renal Biopsy Guidelines of the Renal Pathology S. Practice guidelines for the renal biopsy. Modern Pathology. 2004;17:1555-1563.

19. McAleer S, Fast A, Xue Y, et al. Deep machine learning-assisted multiphoton microscopy to reduce light exposure and expedite imaging. arXiv preprint arXiv.2011.06408. 2020.

20. Kistenev YV, Vrazhnov DA, Nikolaev VV, Sandykova EA, Krivova NA. Analysis of Collagen Spatial Structure Using Multiphoton Microscopy and Machine Learning Methods. Biochemistry (Mosc). 2019;84:S108-s123.

21. Ranjit S, Dobrinskikh E, Montford J, et al. Label-free fluorescence lifetime and second harmonic generation imaging microscopy improves quantification of experimental renal fibrosis. Kidney International. 2016;90:1123-1128.

\section{Figures}

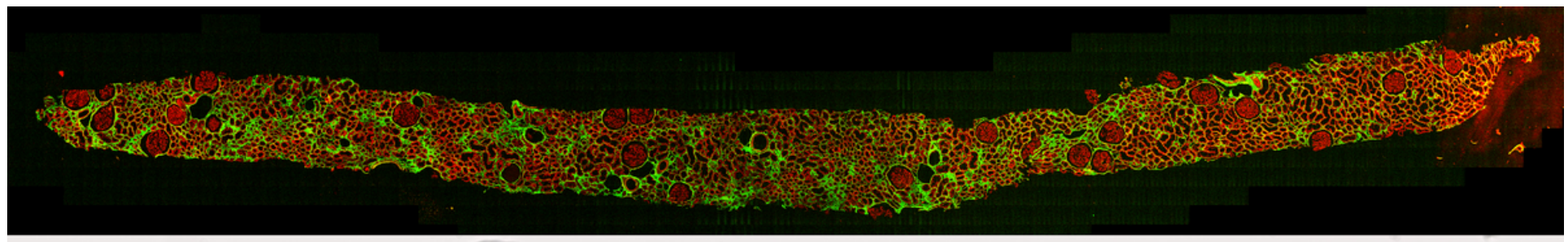

\section{Figure 1}

MPM Imaging of Renal Explant Biopsy (S/N 5) with lowest CART value and Corresponding Hematoxylin and Eosin (H\&E) Image

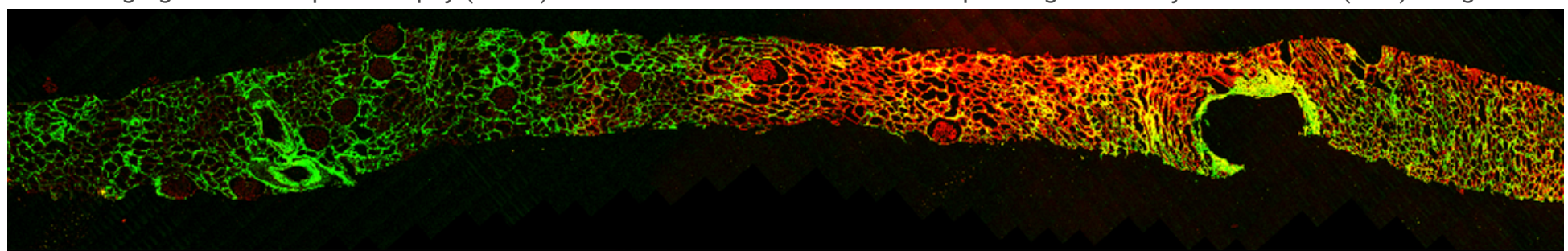

\section{Figure 2}

MPM Imaging of Renal Explant Biopsy (S/N 9) with highest CART value and Corresponding Hematoxylin and Eosin (H\&E) Image 